\title{
Desigualdades da prevalência de hipertensão arterial entre adolescentes brasileiros
}

\author{
Prevalence disparities of hypertension among adolescents in Brazil \\ Desigualdades en la prevalencia de hipertensión arterial entre adolescentes brasileños \\ Bruna Graziela Alcântara de Souza ${ }^{1}$ (D) https://orcid.org/0000-0002-5077-2362 \\ Lorrane Lídia Silva Guimarães ${ }^{1}$ (D) https://orcid.org/0000-0002-6011-8532 \\ Mariana Santos Felisbino-Mendes ${ }^{1}$ (D) https://orcid.org/0000-0001-5321-5708 \\ Sheila Aparecida Ferreira Lachtim ${ }^{1}$ (D) htps://orcid.org/000-0002-3323-5776 \\ Ed Wilson Rodrigues Vieira ${ }^{1}$ (D) https://orcid.org/0000-0001-8198-7270
}

\section{Resumo}

Objetivo: Estimar a prevalência e analisar fatores demográficos e socioeconômicos associados à hipertensão arterial entre adolescentes brasileiros.

Métodos: Trata-se de um estudo transversal, multicêntrico e de abrangência nacional com 4.471 adolescentes que foram entrevistados durante as avaliações externas do Programa Nacional de Melhoria da Qualidade da Atenção Primária, entre 2013 e 2014. Foram estimadas as prevalências de hipertensão, com seus intervalos de 95\% de confiança (IC95\%) segundo fatores socioeconômicos e demográficos. Diferenças entre prevalências foram analisadas pelo teste Qui-quadrado de Pearson. Realizou-se regressão de Poisson com variâncias robustas para estimar os fatores associados à hipertensão.

Resultados: A prevalência nacional de hipertensão foi 4,2\% (IC95\%: 3,6-4,8\%), sem diferenças estatisticamente significativas entre as regiões do país. Dentre os fatores demográficos e socioeconômicos analisados apenas a raça ou cor preta apresentou maior prevalência de diagnóstico de hipertensão (RP: 1,65; IC95\%: 1,07-2,55).

Conclusão: Esses achados evidenciam iniquidade em saúde visto que a cor da pele pode ser considerada como proxy de condição socioeconômica.

\section{Abstract}

Objective: To estimate the prevalence and analyze demographic and socioeconomic factors associated with arterial hypertension among Brazilian adolescents.

Methods: This is a cross-sectional, multicentric and nationwide study with 4,471 adolescents who were interviewed during external evaluations of the National Program for Quality Improvement in Primary Care, between 2013 and 2014. The prevalence of hypertension was estimated, with its 95\% confidence intervals (Cl95\%) according to socioeconomic and demographic factors. Differences between prevalence were analyzed using Pearson's chi-square test. Poisson regression with robust variances was performed to estimate factors associated with hypertension. Results: The national prevalence of hypertension was 4.2\% (CI95\%: 3.6-4.8\%), with no statistically significant differences between the regions of the country. Among the demographic and socioeconomic factors analyzed, only race black had a higher prevalence of diagnosis of hypertension (PR: 1.65; Cl95\%: 1.07-2.55).

Conclusion: These findings show health inequity, as skin color can be considered a proxy for socioeconomic status.

\section{Resumen}

Objetivo: Estimar la prevalencia y analizar los factores demográficos y socioeconómicos asociados a la hipertensión arterial en adolescentes brasileños.

Métodos: Se trata de un estudio transversal, multicéntrico y de ámbito nacional con 4.471 adolescentes que fueron entrevistados durante las evaluaciones externas del Programa Nacional de Mejoramiento de la Calidad en Atención Primaria, entre 2013 y 2014. Se estimó la prevalencia de hipertensión arterial, con su 95\% de intervalos de confianza (IC95\%) según factores socioeconómicos y demográficos. Las diferencias entre las prevalencias se analizaron mediante la prueba de chi-cuadrado de Pearson. Se realizó una regresión de Poisson con varianzas robustas para estimar los factores asociados con la hipertensión.

Resultados: La prevalencia nacional de hipertensión fue de 4.2\% (IC95\%: 3.6-4.8\%), sin diferencias estadísticamente significativas entre las regiones del país. Entre los factores demográficos y socioeconómicos analizados, solo la raza o el color negro tuvieron una mayor prevalencia de diagnóstico de hipertensión (RP: 1,65; IC95\%: 1,07-2,55)

Conclusión: Estos hallazgos muestran inequidad en salud, ya que el color de la piel puede considerarse un indicador del nivel socioeconómico.

\section{Como citar:}

Souza BG, Guimarães LL, Felisbino-Mendes MS, Lachtim SA, Vieira EW. Desigualdades da prevalência de hipertensão arterial entre adolescentes brasileiros. Rev Soc Bras Enferm Ped. 2021;21(2):78-84.
Descritores

Hipertensão; Adolescente; Fatores socioeconômicos; Grupos raciais; Atenção primária à saúde; Enfermagem pediátrica

\section{Keywords}

Hypertension; Adolescent;

Socioeconomic factors; Continental population group; Primary health care; Pediatric nursing

\section{Descriptores}

Hipertensión; Adolescentes; Factores socioeconómicos; Grupos de población continentales; Atención primaria de salud; Enfermería pediátrica

\footnotetext{
${ }^{1}$ Escola de Enfermagem, Universidade Federal de Minas Gerais, Belo Horizonte, MG, Brasil.

Conflitos de interesse: nada a declarar.

Submetido: 23 de Abril de 2021 | Aceito: 14 de Dezembro de 2021

Autor correspondente: Ed Wilson Rodrigues Vieira | E-mail: edwilsonviera@ufmg.br

DOl: http://dx.doi.org/10.31508/1676-379320210012
} 


\section{Introdução}

A hipertensão arterial é a maior causa de anos de vida perdidos ajustados por incapacidades, além de ser um dos principais fatores de risco para o desenvolvimento de morbimortalidade por doenças cardiovasculares em todo o mundo..$^{(1)}$ Sua elevada prevalência na população geral brasileira, cerca de $25 \%$, a posiciona como um dos fatores de risco de maior impacto nos sistemas público e privado de saúde.(2)

Para além dessa importante prevalência, uma variedade de evidências apontam que a hipertensão tem se iniciado ainda na adolescência, ou antes, e progredido ao longo do tempo. ${ }^{(3)}$ Essas evidências indicam que se trata de um processo fisiopatológico ao longo da vida e que os determinantes, já conhecidos para a população adulta, como dieta e atividade física, são também importantes na população juvenil. ${ }^{(4,5)}$ Além disso, indicam que adolescentes com hipertensão poderão apresentar precocemente complicações como aterosclerose coronariana, hipertrofia ventricular esquerda e microalbuminúria, considerados importantes marcadores de risco para mortalidade precoce.

Estudos prévios, desconsiderando-se os aspectos metodológicos, apontam prevalências de hipertensão na população adolescente na ordem de 4 a $20 \%{ }^{(5-7)}$ Por definição, adolescentes são considerados hipertensos quando a pressão arterial sistólica e/ou a pressão arterial diastólica forem superiores ao percentil 95, de acordo com a idade, o sexo e o percentil de altura, em pelo menos três ocasiões distintas. ${ }^{(8)}$

No Brasil, a grande maioria das pesquisas sobre hipertensão entre adolescentes, tem enfocado os fatores de risco antropométricos, como a obesidade e o sobrepeso, por serem os mais consistentemente documentados, desconsiderando, em grande medida, os fatores socioeconômicos e demográficos. Sabe-se que estes podem influenciar o desenvolvimento da hipertensão por estarem intimamente relacionados com os hábitos de vida e consequentemente com as iniquidades em saúde. ${ }^{(9)}$ Além disso, poucos estudos consideram a grande extensão territorial do país ${ }^{(7)}$ e as possíveis desigualdades regionais. Assim, este estudo teve como objetivo estimar a prevalência e analisar fatores demográficos e socioeconômicos associados à hipertensão arterial entre adolescentes brasileiros.

\section{Métodos}

Trata-se de estudo que utilizou dados secundários de um inquérito transversal, multicêntrico e de abrangência nacional, com adolescentes usuários dos serviços de Atenção Primária que participaram do segundo ciclo de avaliações externas do Programa de Melhoria da Qualidade da Atenção Primária (PMAQ-AB), do Ministério da Saúde brasileiro (MS). O PMAQ-AB é um programa de incentivo financeiro que objetiva a melhoria do acesso e da qualidade da atenção primária à saúde no contexto do Sistema Único de Saúde (SUS), constituindo-se de avaliações externas e de indicadores de desempenho. ${ }^{(10)}$

O presente estudo utilizou os dados do segundo ciclo dessas avaliações, ocorrido entre outubro de 2013 e março de 2014. Esse ciclo de avaliações foi conduzido pelo Ministério da Saúde em parceria com 41 instituições federais de ensino e pesquisa lideradas pela Fundação Instituto Oswaldo Cruz (Fiocruz). ${ }^{(10)}$ Apesar dos dados do terceiro ciclo de avaliações externas já estarem disponíveis no momento de preparação deste estudo, optou-se por utilizar apenas os dados do segundo, uma vez que somente esse possuía adolescentes a partir dos 16 anos de idade. Os adolescentes no terceiro ciclo eram relativamente mais velhos, todos com 18 ou 19 anos de idade. ${ }^{(10)}$ Dessa forma optou-se por utilizar apenas os dados do segundo ciclo de avaliações por ter uma amostra com faixa etária mais abrangente entre os adolescentes, sendo considerados para o estudo aqueles indivíduos entre 16 e 19 anos.

O banco de dados utilizado tem acesso aberto e está disponível em: https://aps.saude.gov.br/ape/pmaq/ ciclo2/ e foi acessado em fevereiro de 2019. Por se tratar de um estudo que utiliza dados de domínio público de acesso irrestrito sem a identificação de indivíduos, não foi necessário submeter à apreciação de Comitê de Ética e Pesquisa. Os instrumentos de coleta estavam organizados em três módulos, sendo que neste estudo foram utilizados os dados das entrevistas com usuários que estavam presentes nos serviços no dia das visitas para avaliações externas (questionários, Módulo III).

A coleta envolveu cerca de mil entrevistadores devidamente capacitados para este fim pelas instituições de ensino e pesquisa responsáveis. Os questionários foram aplicados presencialmente utilizando dispositivos eletrônicos - tablets. Para responder aos questionários foram selecionados usuários que não passariam por 
consulta com médico ou enfermeiro no dia da entrevista. Não foram considerados os usuários que não compareciam ao serviço há mais de 12 meses e aqueles que o visitavam pela primeira vez..$^{(10)}$

As respostas ao questionário eram enviadas automaticamente a um servidor central do Ministério da Saúde e a avaliação da consistência dos dados ficou a cargo das instituições de ensino e pesquisa parceiras, sob a coordenação do Departamento de Atenção Primária do Ministério da Saúde. Tal questionário era estruturado e continha dados geográficos, demográficos, socioeconômicos e dados sobre acesso e utilização dos serviços de saúde que deveriam ser respondidas pelo usuário.

Os dados originais contavam com 114.615 usuários de cerca de $95 \%$ das equipes de Atenção Primária do país, sendo selecionados para este estudo, inicialmente, todos os 4.495 adolescentes com idades entre 16 e 19 anos, das 26 Unidades Federativas e do Distrito Federal. Destes, foram excluídos 24 que não sabiam responder se já haviam recebido, ou não, diagnóstico médico de hipertensão (diagnóstico autorreferido). Assim, a amostra estudada contou com 4.471 adolescentes.

O levantamento das informações referentes à hipertensão ocorreu por meio da seguinte pergunta: "algum médico lhe disse que o(a) senhor(a) tem/teve pressão alta (hipertensão)?" Os dados demográficos considerados foram sexo (masculino ou feminino), idade (em anos), e cor ou raça (branca, preta, amarela, parda ou mestiça ou indígena). Os dados socioeconômicos foram: escolaridade ideal para a idade (sim ou não), ser beneficiário ou viver em família beneficiária de auxílio financeiro governamental (Bolsa Família, sim ou não) e trabalhar (sim ou não). Para a escolaridade, assumiu-se que idades entre 16 e 18 anos que informaram Ensino Médio incompleto ou mais estavam na condição de escolaridade ideal para a idade. Para os adolescentes com idades iguais a 19 anos, somente aqueles com Ensino Médio completo ou mais foram considerados na condição de escolaridade ideal para a idade. Como dados geográficos foram consideradas as regiões geopolíticas brasileiras - Norte, Nordeste, Sudeste, Centro-Oeste e Sul.

Os dados, disponibilizados em planilhas do Microsoft Excel ${ }^{\circledR}$, foram transferidos para o software Statistical Package for Social Sciences - SPSS versão 15.0 e a análise estatística foi realizada entre fevereiro de 2019 e abril de 2020. Analisou-se primeiramente as características da população estudada segundo as regiões do país, considerando as frequências absolutas e relativas e o teste qui-quadrado de Pearson. A prevalência de hipertensão foi calculada considerando o número de adolescentes com hipertensão arterial autorreferida dividido pelo número de adolescentes estudados e multiplicado por 100. Diferenças nas prevalências de hipertensão foram analisadas pelo teste qui-quadrado de Pearson e pelo Intervalo de Confiança de 95\% (IC95\%). Utilizou-se a regressão de Poisson com variâncias robustas para estimar a razão de prevalência e seus respectivos IC de 95\% para identificar associações entre as características demográficas, socioeconômicas e geográficas com a ocorrência de hipertensão, individualmente e no modelo multivariado. Para o modelo multivariado foram considerados as variáveis com valor de $p<0,20$ na análise bivariada. Considerou-se o nível de significância estatística de $5 \%$ em todas as análises.

\section{Resultados}

A amostra de adolescentes estudada foi composta por maioria do sexo feminino, variando de $86,0 \%$ na região Sul a 93,7\% na região Centro-Oeste (Tabela 1). Quanto a idade, prevaleceu em todas as regiões, adolescentes entre 18 ou 19 anos. Exceto na região Sul, a cor ou raça parda ou mestiça foi predominante na amostra. De um modo geral, apenas pouco mais da metade dos adolescentes estudados tinha escolaridade ideal para sua idade $(56,1 \%)$, a maioria não trabalhava $(78,8 \%)$ e não era beneficiária ou vivia em família que recebia auxílio financeiro governamental - Programa Bolsa Família (74\%) (Tabela 1). Características descritivas dos adultos jovens (18 a 24 anos de idade) estudados, Brasil, 20172018, segundo regiões brasileiras $(n=17.682)$.

A prevalência geral de hipertensão arterial entre os adolescentes estudados foi de $4,2 \%$, variando, conforme intervalo de confiança de $95 \%$, de 3,6 a 4,8\% (Tabela 2). Observamos diferenças nas prevalências entre as cinco diferentes regiões do país, porém sem significância estatística $(p=0,690)$. Os adolescentes da região Centro-Oeste apresentaram a prevalência mais elevada (5,5\%; IC95\%: 3,4 - 8,5) e aqueles da região Sudeste a mais baixa (3,7\%; IC95\%: 2,8 - 4,9). A análise multivariada, ajustada por idade, raça ou cor, escolaridade ideal para a idade e auxílio financeiro governamental 
Tabela 1. Características sociodemográficas dos adolescentes participantes das avaliações externas do Programa de Melhoria da Qualidade da Atenção Primária (2013-2014)

\begin{tabular}{|c|c|c|c|c|c|c|c|}
\hline $\begin{array}{l}\text { Características } \\
\text { sociodemográficas }\end{array}$ & $\begin{array}{c}\mathrm{SE} \\
\mathrm{n}=1.311 \\
\mathrm{n}(\%)\end{array}$ & $\begin{array}{c}\mathrm{NE} \\
\mathrm{n}=1.686 \\
\mathrm{n}(\%)\end{array}$ & $\begin{array}{c}N \\
n=640 \\
n(\%)\end{array}$ & $\begin{array}{c} \\
n=486 \\
n(\%)\end{array}$ & $\begin{array}{c}\mathrm{C} \\
\mathrm{n}=348 \\
\mathrm{n}(\%)\end{array}$ & $\begin{array}{c}\text { Brasil } \\
\mathrm{n}=4.471 \\
\mathrm{n}(\%)\end{array}$ & $p$-value* \\
\hline Sexo & & & & & & & 0,002 \\
\hline Feminino & $1196(91,2)$ & $1528(90,6)$ & $587(91,7)$ & $418(86,0)$ & $326(93,7)$ & $4055(90,7)$ & \\
\hline Masculino & $115(8,8)$ & $158(9,4)$ & $53(8,3)$ & $68(14,0)$ & $22(6,3)$ & $416(9,3)$ & \\
\hline Idade (em anos) & & & & & & & $<0,001$ \\
\hline 16 a 17 & $228(17,4)$ & $170(10,1)$ & $91(14,2)$ & $29(6,0)$ & $41(11,8)$ & $559(12,5)$ & \\
\hline 18 a 19 & $1083(82,6)$ & $1516(89,9)$ & $549(85,8)$ & $457(94,0)$ & $307(88,2)$ & $3912(87,5)$ & \\
\hline Raça ou cora & & & & & & & $<0,001$ \\
\hline Branca & $414(31,8)$ & $354(21,4)$ & $97(15,4)$ & $316(66,8)$ & $112(32,9)$ & $1293(29,4)$ & \\
\hline Preta & $227(17,4)$ & $236(14,3)$ & $59(9,4)$ & $38(8,0)$ & $31(9,1)$ & $591(13,4)$ & \\
\hline Amarela & $48(3,7)$ & $54(3,3)$ & $30(4,8)$ & $10(2,1)$ & $17(5,0)$ & $159(3,6)$ & \\
\hline Parda/mestiça & $604(46,4)$ & $987(59,7)$ & $422(66,9)$ & $107(22,6)$ & $174(51,2)$ & $2294(52,2)$ & \\
\hline Indígena & $8(0,6)$ & $22(1,3)$ & $23(3,6)$ & $2(0,4)$ & $6(1,8)$ & $61(1,4)$ & \\
\hline Escolaridade/idade ideal ${ }^{b}$ & & & & & & & 0,013 \\
\hline Sim & $784(59,8)$ & $917(54,4)$ & $336(52,5)$ & $272(56,0)$ & $197(56,6)$ & $2506(56,1)$ & \\
\hline Não & $527(40,2)$ & $769(45,6)$ & $304(47,5)$ & $214(44,0)$ & $151(43,4)$ & $1965(43,9)$ & \\
\hline Trabalha & & & & & & & $<0,001$ \\
\hline Sim & $324(24,7)$ & $249(14,8)$ & $75(11,7)$ & $210(43,2)$ & $88(25,3)$ & $946(21,2)$ & \\
\hline Não & $987(75,3)$ & $1437(85,2)$ & $565(88,3)$ & $276(56,8)$ & $260(74,7)$ & $3525(78,8)$ & \\
\hline Bolsa Famíliac $^{c}$ & & & & & & & $<0,001$ \\
\hline Sim & $251(19,2)$ & $577(34,3)$ & $186(29,2)$ & $63(13,1)$ & $82(23,6)$ & $1159(26,0)$ & \\
\hline Não & $1054(80,8)$ & $1105(65,7)$ & $471(70,8)$ & $418(86,9)$ & $265(76,4)$ & $3293(74,0)$ & \\
\hline
\end{tabular}

Tabela 2. Análise não ajustada e ajustada da prevalência de hipertensão arterial entre os adolescentes que participaram das avaliações externas do Programa de Melhoria da Qualidade da Atenção Primária

\begin{tabular}{|c|c|c|c|c|c|c|}
\hline \multicolumn{7}{|c|}{ Hipertensão arterial entre adolescentes } \\
\hline \multirow{2}{*}{$\begin{array}{l}\text { Características } \\
\text { sociodemográficas }\end{array}$} & \multirow{2}{*}{$n(\%)$} & \multirow{2}{*}{ IC 95\% } & \multirow[b]{2}{*}{$p$-value } & \multirow{2}{*}{$\begin{array}{c}\text { Não ajustada } \\
n=4471 \\
R P(I C 95 \%)\end{array}$} & \multicolumn{2}{|c|}{$\begin{array}{c}\text { Ajustada* } \\
n=4320\end{array}$} \\
\hline & & & & & RP(IC95\%) & $p$-value \\
\hline Brasil & $186(4,2)$ & $(3,6-4,8)$ & & & & \\
\hline Regiões & & & 0,690 & & & \\
\hline Sudeste & $49(3,7)$ & $(2,8-4,9)$ & & 1,00 & & \\
\hline Nordeste & $69(4,1)$ & $(3,2-5,2)$ & & $1,09(0,76-1,57)$ & & \\
\hline Norte & $27(4,2)$ & $(2,9-6,2)$ & & $1,13(0,71-1,79)$ & & \\
\hline Sul & $22(4,5)$ & $(2,9-6,9)$ & & $1,21(0,74-1,98)$ & & \\
\hline Centro-oeste & $19(5,5)$ & $(3,4-8,5)$ & & $1,46(0,87-2,45)$ & & \\
\hline Sexo & & & 0,662 & & & \\
\hline Feminino & $146(4,1)$ & $(3,5-4,8)$ & & 1,00 & & \\
\hline Masculino & $19(4,6)$ & $(2,8-7,2)$ & & $1,11(0,70-1,76)$ & & \\
\hline Idade & & & 0,062 & & & \\
\hline $16 \mathrm{e} 17$ & $15(2,7)$ & $(1,6-5,1)$ & & 1,00 & 1,00 & \\
\hline $18 \mathrm{e} 19$ & $171(4,4)$ & $(3,8-5,1)$ & & $1,63(0,97-2,74)$ & $1,50(0,89-2,52)$ & 0,127 \\
\hline Raça ou cor $(n=4.398)$ & & & 0,124 & & & \\
\hline Branca & $45(3,5)$ & $(2,6-4,7)$ & & 1,00 & 1,00 & \\
\hline Parda/mestiça & $93(4,1)$ & $(3,3-5,0)$ & & $1,16(0,82-1,65)$ & $1,17(0,83-1,66)$ & 0,350 \\
\hline Amarela & $9(5,7)$ & $(2,8-10,8)$ & & $1,63(0,81-3,26)$ & $1,67(0,83-3,36)$ & 0,144 \\
\hline Preta & $34(5,8)$ & $(4,1-8,0)$ & & $1,65(1,07-2,55)$ & $1,65(1,07-2,55)$ & 0,023 \\
\hline Indígena & $1(1,6)$ & $(0,1-10,0)$ & & $0,47(0,07-3,36)$ & - & - \\
\hline Escolaridade ideal para idade & & & 0,064 & & & \\
\hline Sim & $92(3,7)$ & $(3,0-4,5)$ & & 1,00 & 1,00 & \\
\hline Não & $94(4,8)$ & $(3,9-5,8)$ & & $1,30(0,98-1,73)$ & $1,26(0,94-1,67)$ & 0,109 \\
\hline Trabalha & & & 0,223 & & & \\
\hline Não & $140(4,0)$ & $(3,4-4,7)$ & & 1,00 & & \\
\hline $\operatorname{Sim}$ & $46(4,9)$ & $(3,6-6,5)$ & & $1,22(0,88-1,69)$ & & \\
\hline Bolsa Família $(\mathrm{n}=4.452)$ & & & 0,051 & & & \\
\hline $\operatorname{Sim}$ & $37(3,2)$ & $(2,3-4,4)$ & & 1,00 & & \\
\hline Não & $149(4,5)$ & $(3,9-5,3)$ & & $1,42(1,00-2,02)$ & $1,37(0,96-1,96)$ & 0,137 \\
\hline
\end{tabular}


familiar, evidenciou que, dentre os fatores estudados, a cor ou raça preta esteve associada à maior prevalência de hipertensão entre os adolescentes brasileiros (Tabela 2). Assim, adolescentes que se autodeclararam da cor ou raça preta apresentaram maior prevalência de hipertensão quando comparados aos adolescentes de cor ou raça branca (RP=1,65; IC95: 1,07 - 2,55).

\section{Discussão}

Os resultados evidenciaram considerável prevalência de hipertensão arterial entre adolescentes brasileiros, porém, sem diferenças estatísticas entre as cinco regiões do país. Essa prevalência corrobora resultados de recente metanálise que apontou uma estimativa global de hipertensão de 4,0\% entre crianças e adolescentes, ${ }^{(11)}$ porém é inferior aos $8,0 \%$ de prevalência em metanálise com adolescentes brasileiros. ${ }^{(5)}$ Mas, comparar a prevalência com outros estudos requer certa cautela, dada a variabilidade de metodologias, técnicas de mensuração e características dos adolescentes estudados, sobretudo em relação à faixa etária.

A prevalência encontrada em pesquisas que consideraram faixa etária semelhante à deste estudo, mas com mensuração direta dos níveis pressóricos, em geral, foi mais elevada. Entre adolescentes com idades entre 14 e 19 anos, foi de 6,4\% $\%^{(12)}$ e com idades entre 15 e 17 anos, foi de 6,9\%.(7) No entanto, mesmo que já aponte para a seriedade da questão, frente ao contexto epidemiológico e de Saúde Pública, essa prevalência pode estar subestimando o número de adolescentes com hipertensão, pois até $75 \%$ daqueles hipertensos podem estar sem diagnóstico. ${ }^{(9)}$

Apesar de não ser um problema de saúde novo, ainda hoje, quando realizado, o diagnóstico de hipertensão nos adolescentes tem sido feito tardiamente e muitos profissionais ainda não seguem as recomendações mais recentes de hipertensão pediátrica. ${ }^{(13)}$ Por consequência, o uso das novas recomendações, somado à realização de diagnósticos precoces, poderiam, presumivelmente, resultar em um aumento relativo da prevalência do diagnóstico entre os adolescentes brasileiros, além de oportunizar cuidados cada vez mais precoces.

Os resultados revelaram também que a prevalência de hipertensão foi maior naqueles adolescen- tes que se autodeclararam de raça ou cor preta. Sobre essa relação, primeiramente, é preciso considerar que é praticamente consensual que a hipertensão é uma doença complexa e multifacetada, com muitos fatores contribuintes. E, embora não haja dúvidas que fatores como dieta e nutrição exerçam importante influência, reconhece-se que os efeitos da predisposição racial são ainda pouco elucidados. ${ }^{(9)}$

Mas, a respeito da maior prevalência de hipertensão entre adolescentes de raça ou cor preta, pode-se afirmar que não se trata de um achado incomum nos estudos epidemiológicos. ${ }^{(14)}$ Com frequência, raça ou cor aparece entre os fatores de risco não modificáveis para a hipertensão, seja na infância ou na adolescência. ${ }^{(9)}$ Entre indivíduos adultos também se encontra maior prevalência de hipertensão entre aqueles de raça ou cor preta. ${ }^{(6,8)}$ Mas, as tentativas de explicação da relação entre cor ou raça e hipertensão são pouco consensuais. Um estudo relaciona essa associação com as diferenças de massa corporal, quando se comparou níveis mais baixos de Índice de Massa Corporal, os indivíduos negros tiveram mais hipertensão do que brancos, mas, em níveis mais altos, os indivíduos brancos tiveram mais hipertensão do que os negros. ${ }^{(15)}$

Outra possibilidade para explicar a associação entre raça ou cor e maior prevalência de hipertensão está ancorada numa possível existência de diferenças fisiológicas inatas entre grupos populacionais. Entretanto, essa é uma hipótese historicamente contestada e pouco fundamentada. Levando-se em conta uma visão mais holística sobre grupos raciais, diferenças sociais e culturais poderiam explicar a maior prevalência de hipertensão entre os adolescentes negros. ${ }^{(16)}$ Prevalências de doenças crônicas não transmissíveis e de seus fatores de risco são mais elevadas em indivíduos com alguma vulnerabilidade social, incluindo a raça. ${ }^{(9)}$ Assim, esse resultado para a cor ou raça dos adolescentes poderia ser explicado considerando-a como uma variável proxy das vulnerabilidades sociais brasileiras. Cabe ressaltar que outros fatores investigados neste estudo, que igualmente podem determinar vulnerabilidades sociais, também apresentaram gradientes maiores de hipertensão quando comparados a situações melhores, apesar de não terem sido significativos do ponto de vista estatístico.

Corroborando a compreensão do complexo fenômeno observado nesta investigação, estudo conduzido no Brasil sobre as causas da hipertensão demons- 
trou que não há diferença nos valores pressóricos entre crianças pré-púberes negras e não negras ${ }^{(17)} \mathrm{Em}$ bora tenha observado diferença fisiológica em relação a rigidez arterial, que está relacionada a elevação da pressão arterial, isso pode indicar a necessidade de compreender melhor os determinantes sociais relacionados às iniquidades nas doenças crônicas, em especial as iniquidades na hipertensão.

É importante destacar que, de fato, a população negra compõe a maioria entre os moradores periféricos, os trabalhadores subempregados e sem proteção no emprego. Já os jovens negros estão entre as maiores vítimas de violência e suicídio na atualidade. ${ }^{(18)}$ Nesse sentido, é relevante observar que os desfechos mais graves da hipertensão, como insuficiência renal e acidente vascular cerebral, têm impactos ainda maiores na população negra, uma vez que se acumulam aos piores indicadores de saúde-doença às precárias condições de vida dessa população, como dificuldade de acesso aos serviços de saúde e educação e consumo. Chama atenção o fato de que as desigualdades sociais em saúde não são apenas reflexo de diferentes escolhas pessoais que levam a distintos modos saudáveis ou não de viver a vida, senão desigualdades injustas decorrentes de alguma forma de injustiça social.(18)

Uma das limitações do presente estudo é que, dada a sua abordagem transversal, o presente estudo não fornece inferências sobre a relação causa e efeito. Mesmo não sendo uma amostra representativa da população adolescente brasileira, pode representar o conjunto de adolescentes que utiliza os serviços de saúde de Atenção Primária. De fato, as características da amostra assemelham-se àquelas da população que mais frequenta esses serviços, inclusive com uma proporção muito maior de mulheres. Mas ressalta-se que a prevalência de hipertensão não foi diferente entre os sexos. Os adolescentes estudados pertencem provavelmente ao mesmo grupo social homogêneo, o que pode explicar porque esta investigação não captou diferenças em outros fatores demográficos e socioeconômicos. ${ }^{(19)}$ Então, apesar dessa particularidade amostral, esse estudo fornece informações valiosas sobre o que esperar de hipertensão em adolescentes vivendo no território de abrangência das unidades básicas de saúde. Outra limitação é o uso de diagnósticos autorreferidos para se estudar a hipertensão, ainda que seja uma estratégia de pesquisa internacionalmente respeitada, pode resultar em subestimação da população afetada. ${ }^{(20)}$

Os resultados, mesmo com as limitações assumidas pela escolha metodológica, são importantes de serem considerados por profissionais de saúde que acompanham adolescentes na Atenção Primária. A prevalência de doenças é um importante determinante para a vigilância em saúde, e a compreensão das taxas das doenças mais prevalentes, como a hipertensão, deve ser um guia primordial para planejar os serviços de saúde.

Faz-se necessário considerar a probabilidade de hipertensão para desenvolverem ações de prevenção, diagnóstico, tratamento e acompanhamento dos casos, destacando-se o papel do enfermeiro na Atenção Primária no acolhimento e acompanhamento dos adolescentes, sendo importante não se perder a oportunidade de cuidado quando esses indivíduos comparecem ao serviço.

Reforça-se que a pressão arterial deve ser avaliada nas visitas de rotina para a manutenção da saúde do adolescente, com atenção especial à maior prevalência de hipertensão entre os de cor ou raça preta. Ressalta-se a importância de envolvimento dos responsáveis pelas políticas públicas, no desenvolvimento de ações nacionais para melhorar a identificação e tratamento da hipertensão na adolescência. Destaca-se, ainda, que o PMAQ-AB pode ser um importante instrumento para detecção, monitoramento e vigilância em saúde.

\section{Conclusão}

Cerca de quatro em cada 100 adolescentes brasileiros atendidos nos serviços de Atenção Primária possuem hipertensão arterial, mas essa prevalência pode estar subestimada. Aqueles de raça ou cor preta apresentam maior prevalência desse agravo, evidenciando iniquidade em saúde, ou seja, pior condição de saúde daqueles que já possuem uma vulnerabilidade social, aqui considerando a cor da pele como proxy de condição socioeconômica.

\section{Agradecimentos}

À Pró-reitoria de Pesquisa da Universidade Federal de Minas Gerais. 


\section{Contribuições}

Souza BGA trabalhou na análise e interpretação dos dados, na redação do artigo e na aprovação da versão final. Felisbino-Mendes MS trabalhou na análise e interpretação dos dados, na revisão crítica do artigo e na aprovação da versão final. Lachtim SAF trabalhou na análise e interpretação dos dados, na revisão crítica do artigo e na aprovação da versão final. Vieira EWR e Guimarães LSS trabalharam na concepção, análise e interpretação dos dados, na redação e revisão crítica do artigo e na aprovação da versão final.

\section{Referências}

1. Global Burden of Disease Study 2017. Global Burden of Disease Study 2017. Lancet 2017;5:1-27.

2. Brasil. Ministério da Saúde. Vigitel Brasil 2019: vigilância de fatores de risco e proteção para doenças crônicas por inquerito telefônico [Internet]. Brasilia (DF): Ministério da Saúde; 2020. 137 p [citado 2021 Out 20]. Disponivel em: https://bvsms.saude.gov.br/ bvs/publicacoes/vigitel_brasil_2019_vigilancia_fatores_risco.pdf

3. Oikonen M, Nuotio J, Magnussen CG, Viikari JS, Taittonen L, Laitinen T, et al. Repeated Blood Pressure Measurements in Childhood in Prediction of Hypertension in Adulthood. Hypertension. 2016;67(1):41-7.

4. Andrade GN, Matoso LF, Miranda JW, Lima TF, Gazzinelli A, Vieira EW. Anthropometric indicators associated with high blood pressure in children living in urban and rural areas. Rev Latatino-Am Enfermagem. 2019;27:e3150.

5. Gonçalves VS, Galvão TF, Andrade KR, Dutra ES, Bertolin MN, de Carvalho KM, et al. Prevalence of hypertension among adolescents: Systematic review and meta-analysis. Rev Saude Publica. 2016;50:1-12.

6. Alves RF, Faerstein E. Educational inequalities in hypertension: Complex patterns in intersections with gender and race in Brazil. Int J Equity Health. 2016;15(1):1-9.
7. Bloch KV, Klein CH, Szklo M, Kuschnir MC, Azevedo AG, Barufaldi LA, et al. ERICA: Prevalences of hypertension and obesity in Brazilian adolescents. Rev Saude Publica. 2016;50(supl 1):1s-12s.

8. Malachias MV, Souza W, Plavnik F, Rodrigues C, Brandão A, Neves M, et al. 7a diretriz brasileira de hipertensão arterial. Arq Bras Cardiol. 2016;107(3):103.

9. Ewald DR, Haldeman LA. Risk Factors in Adolescent Hypertension. Glob Pediatr Heal. 2016;3:2333794X1562515.

10. Brasil. Ministério da Saúde. Nota Metodológica da Certificação das Equipes de Saúde Básica [Internet]. Brasilia (DF): Ministério da Saúde; 2015 [citado 2021 Out 20]. Disponível em: http://189.28.128.100/dab/docs/portaldab/documentos/nota_metodologica_2_ ciclo_pmaq.pdf

11. Song P, Zhang Y, Yu J, Zha M, Zhu Y, Rahimi K, et al. Global Prevalence of Hypertension in Children: A Systematic Review and Meta-analysis. JAMA Pediatr. 2019;173(12):115463.

12. Silva A0, Silva MV, Pereira LK, Feitosa WM, Ritti-Dias RM, Diniz PR, et al.Association between general and abdominal obesity with high blood pressure: difference between genders. J Pediatr (Rio J). 2016;92(2):174-80.

13. Sociedade Brasileira de Pediatria. Hipertensão arterial na infância e adolescência; 2019. $25 \mathrm{p}$.

14. Cheung EL, Bell CS, Samuel JP, Poffenbarger T, Redwine KM, Samuels JA. Race and obesity in adolescent hypertension. Pediatrics. 2017;139(5): e20161433.

15. Rosner B, Prineas R, Daniels SR, Loggie J. Blood pressure differences between Blacks and Whites in relation to body size among US children and adolescents. Am J Epidemiol. 2000;151(10):1007-19.

16. Ski CF, Thompson DR, Fitzsimons D, King-Shier K. Why is ethnicity important in cardiovascular care? Eur J Cardiovasc Nurs. 2018;17(4):294-6.

17. Zaniqueli D,Alvim RO, Luiz SG, Oliosa PR, Cunha RS, Mill JG. Ethnicity and arterial stiffness in children and adolescents from a Brazilian population. J Hypertens. 2017;35(11):2257-61.

18. Brasil. Ministério da Saúde. Política Nacional de Saúde Integral da População Negra [Internet]. Brasilia (DF): Ministério da Saúde; 2017.44 p. [citado 2021 Out 20]. Disponível em: https://bvsms.saude.gov.br/bvs/publicacoes/politica_nacional_saude_populacao_ negra_3d.pdf

19. Guibu IA, de Moraes JC, Guerra Junior AA, Costa EA,Acurcio FA, Costa KS, et al. Características principais dos usuários dos serviços de atenção primária à saúde no Brasil. Rev Saude Publica. 2017;51(Supl 2:17s):1s-13s.

20. Goncalves VS, Galvao TF, Silva MT, Kuschnir MC, Dutra ES, Carvalho KM. Accuracy of selfreported hypertension in Brazilian adolescents: Analysis of the Study of Cardiovascular Risk in Adolescents. J Clin Hypertens (Greenwich). 2018;20(4):739-47. 